\title{
Protée
}

\section{Des figures de manipulation dans la création numérique}

\section{Serge Bouchardon}

Volume 39, numéro 1, printemps 2011

Esthétiques numériques. Textes, structures, figures

URI : https://id.erudit.org/iderudit/1006725ar

DOI : https://doi.org/10.7202/1006725ar

Aller au sommaire du numéro

Éditeur(s)

Département des arts et lettres - Université du Québec à Chicoutimi

ISSN

0300-3523 (imprimé)

1708-2307 (numérique)

Découvrir la revue

Citer cet article

Bouchardon, S. (2011). Des figures de manipulation dans la création numérique. Protée, 39(1), 37-46. https://doi.org/10.7202/1006725ar

\section{Résumé de l'article}

Les créations numériques en ligne, qu'il s'agisse de bannières publicitaires, de littérature ou d'art numériques, reposent souvent sur des manipulations de la part du lecteur (par exemple déplacer un élément à l'écran, activer un lien, entrer du texte au clavier). Nous manquons néanmoins d'outils, notamment sémiotiques et sémio-rhétoriques, pour analyser le rôle de ces gestes de manipulation dans la construction du sens. Dans cet article, nous proposons un modèle d'analyse en cinq niveaux. Ce modèle distingue notamment des unités sémiotiques de manipulation et des couplages média. Ces couplages donnent naissance à des figures que nous appelons figures de manipulation. 


\section{DES FIGURES DE MANIPULATION DANS LA CRÉATION NUMÉRIQUE}

SERGE BOUCHARDON

\section{DU MANIPULABLE DANS LES CRÉATIONS NUMÉRIQUES}

À la fin des années 1990, les bannières publicitaires en ligne reposaient essentiellement sur l'animation. Or, depuis plusieurs années, on peut relever un nombre croissant de publicités en ligne qui reposent également sur une manipulation de la part de l'utilisateur. Il peut s'agir de déplacer un élément à l'écran ${ }^{1}$, d'activer un lien ${ }^{2}$, voire d'entrer du texte au clavier ${ }^{3}$. Dans tous ces cas, on peut qualifier ces bannières d'interactives, qu'il s'agisse d'appréhender un contenu existant, d'activer un autre contenu ou de créer un nouveau contenu en introduisant des données (Bouchardon, 2009). Dans le domaine de la création numérique littéraire et artistique, les œuvres interactives existent quant à elles depuis plusieurs décennies.

Des manipulations sont ainsi requises de la part du lecteur pour qu'une création dite interactive puisse se dérouler. Ces manipulations, dans ces créations interactives, ne semblent pas fondamentalement nouvelles. Il existe notamment de nombreux exemples d'œuvres littéraires qui requièrent des interventions physiques de la part du lecteur, à commencer par les Cent mille milliards de poèmes de Raymond Queneau (1961). Espen Aarseth a avancé le terme de «littérature ergodique» pour les désigner: «en littérature ergodique, un effort non trivial est requis du lecteur pour qu'il traverse le texte» (1997: 1; notre traduction [NT]). Ce qui peut néanmoins paraître quelque peu nouveau dans la création numérique interactive, c'est la dimension manipulable du texte lui-même, et non pas seulement du support ou du dispositif. Le texte numérique, autant que d'être un texte donné à lire, peut être un texte donné à manipuler (Ghitalla et Boullier, 2004). Dans certains cas, cette manipulabilité peut même primer la lecture (Saemmer, 2008). Cette dimension manipulable du texte, mais aussi de l'ensemble des formes sémiotiques, ouvre un large champ de possibles dans les créations interactives.

Nous manquons néanmoins d'outils, notamment sémiotiques, pour les analyser. L'objectif de cet article est donc de proposer des outils d'analyse sémiotique - et notamment sémio-rhétorique - de la manipulation dans les créations numériques. Nous limiterons notre champ d'étude aux créations en ligne, qu'il s'agisse du domaine de la création publicitaire ou de celui de la création littéraire et artistique.

Pour cela, nous allons tout d'abord nous intéresser au geste de manipulation dans les créations numériques. Nous proposerons ensuite un modèle d'analyse de la manipulation en cinq niveaux. En nous fondant sur cette approche, nous terminerons par l'étude d'une figure de manipulation dans une création numérique. 
Précisons que cet article doit beaucoup à une réflexion collective, dans le cadre d'un groupe de recherche ${ }^{4}$ - composé d'Alexandra Saemmer, de Philippe Bootz, de Jean Clément et de moi-même sur la sémiotique et la rhétorique des créations numériques.

\section{LE GESTE DE MANIPULATION}

DANS LES CRÉATIONS NUMÉRIQUES

1.1 Le rôle du geste

Dans un article intitulé «Une esthétique de la matérialité», en nous appuyant sur différents exemples (texte mis en mouvement par le lecteur, texte actable, texte-matériau, texte composant un espace de déplacement), nous avancions que «la matérialité du texte est indissociable de l'action du lecteur. [...] C'est le geste du lecteur qui révèle la matérialité du texte" (Bouchardon, 2008: 138). Le geste et le texte manipulable ne peuvent venir à exister que parce qu'ils sont en relation: la relation co-constitue le geste et le texte manipulable.

Pour pouvoir analyser la dimension manipulable dans les créations numériques, il s'agit ainsi de penser le geste ni uniquement à partir du geste en soi, ni uniquement à partir de ce qui se passe à l'écran. Dans la manipulation, le geste prend sens dans l'interaction. C'est cette position que l'on retrouve notamment dans la notion de "geste interfacé» de Jean-Louis Weissberg (2006). Dans une œuvre interactive, le geste acquiert ainsi un rôle particulier, contribuant pleinement à la construction du sens. Yves Jeanneret rappelle que le fait de tourner la page d'un livre «ne suppose a priori aucune interprétation particulière du texte» (2000: 112); dans une ouvre interactive ${ }^{5}$ en revanche, «le fait de cliquer sur un mot (un "hypermot") ou sur un pictogramme (une "icône") est, en lui-même, un acte d'interprétation» (ibid.: 113). Le geste interactif consiste avant tout en une «interprétation actualisée dans un geste» (ibid.: 121).

\subsection{Un répertoire de gestes}

Dans la manipulation de créations interactives en ligne, nous avons affaire à des gestes différents: certes cliquer, double-cliquer, cliquer droit, déplacer la souris (ou bouger le doigt sur le touchpad), laisser le doigt appuyé, relâcher le doigt, taper sur une touche du clavier, mais aussi parfois souffler dans un micro, bouger la tête devant une webcam...

Nous aurions besoin ici d'un répertoire de gestes (une gestique) qui apparaîtraient comme des unités distinctives. Nous sommes néanmoins confrontés à une difficulté: ces gestes de manipulation d'un contenu numérique sont en constante évolution et dépendent des dispositifs. De ce point de vue, la wiimote a fait considérablement évoluer le répertoire de gestes à la disposition d'un joueur. Mais sans même parler de jeux vidéo sur console ou de dispositifs de réalité virtuelle dans les installations numériques, le simple fait de pouvoir utiliser deux doigts pour zoomer/dézoomer avec le trackpad des derniers ordinateurs Macintosh offre des possibilités inédites de manipulation dans les créations en ligne.

Dans d'autres dispositifs, le répertoire de gestes sera bien évidemment plus étendu qu'avec le seul input souris-clavier. Il en est ainsi des gestes de manipulation sur des tables interactives (voir Wobbrock, Ringel Morris et Wilson, 2009), ou encore de l'interaction avec un bureau tactile 3D 6 . Mais dans la mesure où nous limitons notre étude à l'analyse des créations en ligne, nous allons nous intéresser avant tout aux interfaces d'entrée que sont la souris et le clavier, tout en sachant que certains sites Web proposent dès à présent une navigation faisant appel à d'autres interfaces d'entrée que le clavier et la souris, comme la webcam $^{7}$, le micro ${ }^{8}$, voire n'importe quel objet du quotidien dans les expériences de réalité augmentée (par exemple une boîte de céréales au chocolat $^{9}$.

Si l'on veut s'abstraire de la dimension contextuelle des évolutions techniques, on pourrait se poser la question suivante: en tant qu'unité distinctive, le geste peut-il également être considéré comme une unité signifiante, indépendamment du contexte dans lequel il s'insère ou de la ressource média sur laquelle il s'applique? Nous souhaitons avancer ici qu'il y aura des attentes liées à un certain geste effectué dans un certain contexte. Le contexte recrée une situation déjà rencontrée et dans laquelle un geste donné a eu un résultat pertinent. C'est donc le geste en contexte qui est lié à une attente, et non 
le geste lui-même: l'attente est différente lorsqu'on clique pour valider un choix ou lorsqu'on clique sur un lien (Dallet, 1996). Ces attentes sont en partie le fruit de la construction de conventions. Ainsi, le survoler/quitter (roll over roll out), qui permet de faire apparaître/disparaître une ressource média, peut susciter l'attente d'un dévoilement (Bouchardon, 2007). De même qu'il y aurait des traits signifiants possibles liés au mouvement dans l'animation ${ }^{10}$, de même on pourrait parler de traits signifiants possibles liés au geste de manipulation en contexte.

\subsection{Geste et action}

Nous distinguons entre le geste pris comme activité unitaire (appui sur une touche du clavier ou sur le bouton de la souris, déplacement élémentaire de la souris, etc.) et l'action considérée comme un énoncé de gestes (par exemple le glisser/déposer - drag and drop). Le geste, en tant qu'activité unitaire, est lié à une interface matérielle. L'action a quant à elle une signification plus globale liée à un double couplage à un contexte et à un processus. Bien souvent, le média pertinent (avec lequel le geste interagit) est en effet un processus et non son état final. Plus encore que l'animation, la manipulation fait intervenir ce que Philippe Bootz appelle la "profondeur du dispositif» (2004): dans la manipulation, les signes sont fondamentalement "duaux», mettant en œuvre des éléments se situant à la fois du côté du programme et du côté de l'écran.

Forts de cette approche du geste, proposons à présent un modèle d'analyse du geste de manipulation dans les créations numériques en ligne.

\section{L'ANALYSE DU GESTE DE MANIPULATION}

\subsection{Les cinq niveaux d'analyse de la manipulation}

Nous proposons ici une terminologie permettant de distinguer cinq niveaux d'articulation du signe, correspondant à cinq niveaux d'analyse.

\section{Niveau 1. Le gestème}

C'est le premier niveau d'articulation, le niveau le plus bas. Il est le résultat d'un couplage entre une activité physique et une interface d'entrée (par exemple le fait de déplacer la souris ${ }^{11}$ ou d'appuyer sur une touche du clavier). Le gestème correspond à une unité sémiotique distinctive.

Niveau 2. L'actème

L'actème est fabriqué à partir des gestèmes et est le résultat d'un couplage entre le gestème et le processus sur lequel porte la manipulation. Il existe trois types d'actèmes (qui se distinguent par le couplage entre la séquence de gestes et le processus manipulé):

- Actionneur (changement d'état). Par exemple, je clique sur un bouton ou sur un lien hypertexte à l'écran.

- Paramétreur (processus paramétré). Par exemple, j'utilise un ascenseur, à savoir une barre de défilement horizontal ou vertical.

- Perturbateur (processus cogéré). À un certain moment, le programme prend le dessus et fait quelque chose d'incompatible avec le guidage de l'action. C'est par exemple le cas dans Le Rabot poète de Philippe Bootz (2005) ou dans certaines créations de Rafael Rozendaal ${ }^{12}$ qui jouent sur le décalage entre la manipulation de la souris et le déplacement du pointeur à l'écran (visibilité, apparence, vitesse, sens, direction).

Niveau 3. L'unité sémiotique de manipulation (USM)

Les actèmes composent des unités sémiotiques de manipulation. Par exemple, les actèmes "cliquer", «glisser» et «relâcher» peuvent composer l'USM tirer-relâcher. Nous proposons à la fin de cette partie un tableau des USM. Jean-Marie Klinkenberg rappelle que les icônes sont des signes «motivés par ressemblance" (2000: 193). Dans l'icône, quelque chose du monde physique est reconnu comme tel. De même, l'USM fait penser à des actions dans le monde physique. Par exemple, l'USM gratter peut faire penser à l'action de raboter une surface. L'USM est ainsi iconique de situations de manipulation de la vie courante. Elle porte des traits d'iconicité.

\section{Niveau 4. Le couplage média}

Il est question ici du geste interfacique qui résulte du couplage entre l'unité sémiotique de manipulation et l'état média environnant. L'unité sémiotique de manipulation, nous l'avons vu, présente souvent une 
dimension iconique. C'est néanmoins seulement dans son couplage avec des médias que des traits signifiants vont être ou non actualisés. Ceux-ci sont actualisés en fonction du texte, de l'image ou du son sur lequel le geste est appliqué, ainsi qu'en fonction du contexte multimédia et de l'environnement culturel du lecteur.

Plus le champ d'intersection entre les traits signifiants de l'USM et ceux des médias sur lesquels elle s'applique est étendu (et plus l'union des traits mobilisés répond aux attentes du lecteur provenant $\mathrm{du}$ contexte immédiat et des habitudes de lecture), plus la construction du sens relève de ce que l'on pourrait appeler un couplage conventionnel ${ }^{13}$. Lorsque le champ d'intersection est réduit entre les traits signifiants de l'USM et ceux des médias, un différentiel se crée entre les attentes du lecteur et l'état réalisé par la manipulation: on peut parler alors de couplage non conventionnel (par exemple, le geste de cliquer sur un lien n'entraîne aucune activation, ou bien une activation différée, ou encore l'activation d'une multiplication d'éléments).

Il existe trois types de couplage:

- simultané (couplage simultané entre une USM et des médias);

- consécutif (couplage entre une USM et les médias qui précèdent ou suivent);

- différé (couplage entre une USM et un résultat média très éloigné dans le temps) ${ }^{14}$.

Tout couplage entre une USM et des médias est par nature "pluricode», au sens où il fait intervenir plusieurs «sous-énoncés relevant chacun d'un code différent» (Klinkenberg, 2000: 232). Ces couplages média donnent naissance à des figures. Par l'expression «figure de manipulation", nous désignons une relation entre un geste et des médias dans laquelle la construction du sens est fondée sur des processus d'intersection de traits signifiants associés au geste, au média et au contexte. Tout couplage pluricode est une figure, mais toute figure ${ }^{15}$ n'est pas nécessairement un trope (telle la métaphore).

Niveau 5. Le discours interactif

Ce niveau est celui d'une séquence interactive complète de couplages média. Nous sommes ici sur le plan du discours. C'est en effet souvent en prenant en compte l'ensemble du discours interactif que le geste de manipulation prend tout son sens.

\subsection{Analyse d'un exemple}

Illustrons ces cinq niveaux d'articulation du signe par l'analyse d'un exemple publicitaire. Sur le site bannerblog.com est archivée une publicité pour la société "Amanco». Sont représentées des toilettes (voir la figure 1 , reproduction de gauche). Le format vertical de la publicité renforce l'impression d'une pièce exiguë. Il s'agit pour l'internaute de tirer la chasse d'eau. Sur la poignée est écrit le mot «pull» («tirer»). La chasse est animée d'un mouvement de haut en bas qui doit inciter l'internaute à tirer. L'internaute doit tirer la souris vers lui en maintenant le bouton enfoncé: ce geste mime le fait de tirer la poignée de la chasse vers le bas.

La poignée une fois tirée, un violon et des mains apparaissent du fond de la cuvette (voir la figure 1, reproduction du centre). Une musique jouée au violon se fait entendre. Le slogan s'affiche alors: «not every sound deserves to be heard» («les sons ne méritent pas tous d'être entendus»). Puis apparaît le nom du produit: «Silentium PVC soundproof pipes» («conduits insonorisés Silentium en PVC»; voir la figure 1 , reproduction de droite). Si l'internaute tire de nouveau sur la poignée de la chasse, un autre instrument apparaît du fond de la cuvette. Au total, trois instruments (violon, saxophone, accordéon) se succèdent.

Premier niveau: le gestème

L'internaute appuie sur le bouton de sa souris (après avoir positionné le curseur sur le mot «pull»), le maintient appuyé en déplaçant sa souris puis relâche le bouton. Nous avons ici plusieurs gestèmes à l'œuvre dans cette manipulation: l'appui sur le bouton, le mouvement de déplacement, le relâché (appui inversé).

\section{Deuxième niveau: l'actème}

L'actème correspond à une séquence de gestèmes. Il y a ici deux actèmes: un paramétreur (je tire) et un actionneur (je relâche), qui déclenche en même temps 

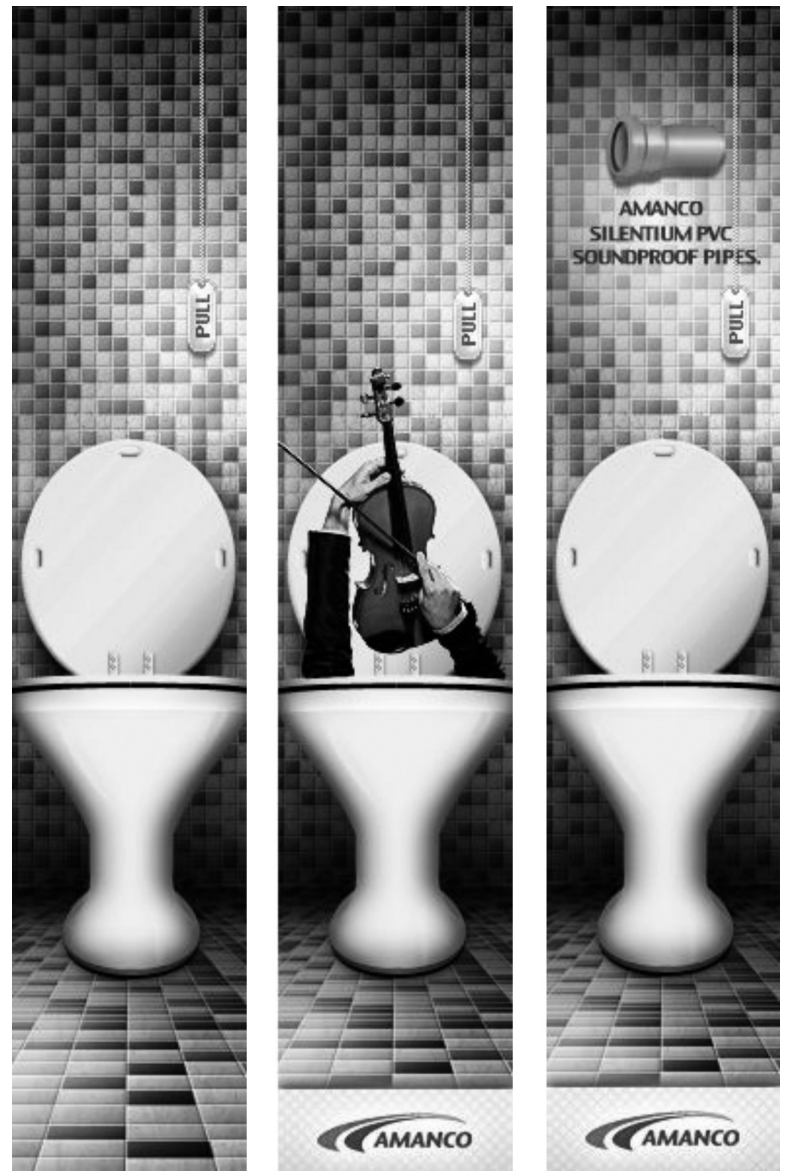

Figure 1

Les trois étapes de la bannière publicitaire en ligne pour la société Amanco (2009): http://www.bannerblog.com.au/2009/09/toilet.php

l'apparition de l'instrument de musique et du son associé.

Troisième niveau:

l'unité sémiotique de manipulation (USM)

Les deux actèmes constituent une seule unité sémiotique de manipulation: tirer-relâcher (voir le tableau des USM à la page suivante).

Il est intéressant de noter ici la dimension iconique de l'USM. L'internaute est incité à faire avec sa souris un geste qui ressemble au geste qu'il est amené à faire quand il tire une chasse d'eau.

Quatrième niveau: le couplage média

On peut relever deux couplages média (l'un simultané, l'autre consécutif):
- le couplage de l'USM tirer-relâcher avec "pull» et avec l'image d'une chasse d'eau (couplage conventionnel);

- le couplage de l'USM tirer-relâcher avec l'apparition du violon en fonction du contexte média, en l'occurrence la cuvette des toilettes (couplage non conventionnel).

Ce second couplage prend en compte l'état initial, le geste de manipulation et l'état final. Dans l'état final, un violon apparaît au lieu de l'eau après qu'on a tiré la chasse d'eau. Cette apparition du violon dans la cuvette des toilettes est incongrue du point de vue du média, mais peut-être également du point de vue du geste interactif: je tire vers le bas et quelque chose surgit de bas en haut (en l'occurrence un violon). L'isotopie générale (cuvette des toilettes, exiguité de l'espace) renforce les couplages média.

Cinquième niveau: le discours interactif

Quand l'internaute a tiré la chasse une première fois, un état attendu est créé: tirer la chasse d'eau fait apparaitre un violon. On peut parler de perturbation de l'attente lorsque c'est un autre instrument qui apparaît la deuxième fois (un saxophone), puis la troisième (un accordéon). L'actionneur du niveau 2 est alors réinterprété comme un perturbateur. L'utilisateur peut penser qu'il y a un tirage aléatoire entre différents instruments. Pourtant, s'il continue à actionner la chasse d'eau, il s'aperçoit que la séquence violon - saxophone - accordéon est toujours la même. Il peut enfin réinterpréter le perturbateur comme un paramétreur.

La force de cette publicité est d'inciter l'utilisateur, de lui-même, à la rejouer pour découvrir si ses attentes seront ou non vérifiées.

Nous avons mentionné dans le troisième niveau que les actèmes en jeu constituaient une seule unité sémiotique de manipulation (tirer-relâcher). Penchonsnous à présent plus précisément sur ces unités sémiotiques de manipulation.

2.3 Tableau des unités sémiotiques de manipulation (USM)

Le tableau ci-dessous propose une typologie

d'unités sémiotiques de manipulation s'appuyant sur 
TABLEAU DES UNTÉS SÉMIOTIQUES DE MANIPULATION (USM)

\begin{tabular}{|c|c|c|c|c|}
\hline USM & Description (actèmes) & $\begin{array}{l}\text { Délimitée } \\
\text { dans le } \\
\text { temps }\end{array}$ & Répétitive & $\begin{array}{l}\text { Traits d'iconicité potentiels } \\
\text { (ce à quoi l'USM fait penser dans le monde } \\
\text { physique) }\end{array}$ \\
\hline Activer & 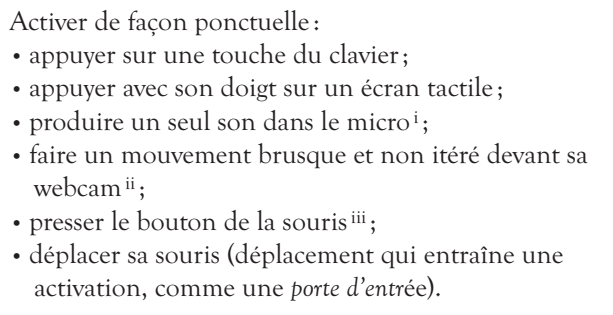 & Oui & Non & $\begin{array}{l}\text { - par un geste, déclencher ou démarrer une } \\
\text { action, la bloquer }{ }^{\text {iv }} \text { ou la stopperv } \\
\text { - pointer dans telle direction }{ }^{\mathrm{vi}} \text {, pénétrer en } \\
\text { un seul pas (et non pas progressivement); } \\
\text { - substituer, remplacer }{ }^{\text {vii }} \text { un élément par un } \\
\text { autre en un seul geste; } \\
\text { - choisir, sélectionner }{ }^{\text {viii... }}\end{array}$ \\
\hline Mouvoir & $\begin{array}{l}\text { - déplacer sa souris dans le plan (survoler); } \\
\text { - déplacer sa souris en laissant une trace (tracer); } \\
\text { - déplacer la main sur un écran tactile; } \\
\text { - déplacer le doigt sur le touchpad dans un mouvement } \\
\text { continu; } \\
\text { - déplacer le stylo sur un agenda numérique. }\end{array}$ & Non & Non & $\begin{array}{l}\text { - caresser ix (un être humain, un animal, une } \\
\text { surface); } \\
\text { - étaler de la matière (des cartes, une crème), } \\
\text { enlever de la poussière; } \\
\text { - dévoiler }{ }^{\mathrm{x}} \text {, révéler }{ }^{\mathrm{xi}}, \text { cacher; } \text {, } \\
\text { - tracer, dessiner }{ }^{\mathrm{xii}}, \text { effacer; } \\
\text { - accélérer }{ }^{\text {xiii, }} \text {, ralentir... }\end{array}$ \\
\hline Gratter & $\begin{array}{l}\text { Déplacer sa souris de façon répétitive (gratter de } \\
\text { façon linéaire - par exemple raboter de façon } \\
\text { unidirectionnelle -, touiller avec des courbes...). }\end{array}$ & Non & Oui & $\begin{array}{l}\text { - raboter une surface, sculpter une matière; } \\
\text { - gratter la peau, gratter un jeu de grattage, } \\
\text { gratter une vitre; } \\
\text { - creuser un trou; } \\
\text { - mélanger des substances, des matières... }\end{array}$ \\
\hline Tirer & $\begin{array}{l}\text { Faire glisser un élément, à la souris (appuyer - tirer) ou } \\
\text { au clavier. }\end{array}$ & Oui & Non & $\begin{array}{l}\text { - déplacer un objet avec prise sur lui xiv (tirer } \\
\text { un rideau, écarter des voiles, tirer une } \\
\text { chasse d'eau, enlever une étiquette, retirer } \\
\text { une enveloppe, remonter la couverture, } \\
\text { ouvrir une boîte de sardines, retirer un } \\
\text { vêtement); } \\
\text { - immerger }\end{array}$ \\
\hline $\begin{array}{l}\text { Tirer- } \\
\text { relâcher }\end{array}$ & $\begin{array}{l}\text { Faire glisser un élément et le relâcher (appuyer - tirer - } \\
\text { relâcher). }\end{array}$ & Oui & Non & $\begin{array}{l}\text { - ordonner, mettre des objets dans les } \\
\text { bonnes cases, ranger des tiroirs ou une } \\
\text { chambre; } \\
\text { - réorganiser, recomposer }{ }^{\mathrm{xvi}} \text { un tableau, faire } \\
\text { un jeu de puzzle; } \\
\text { - déformer }{ }^{\text {xvii }} \text {; } \\
\text { - déclencher un mécanisme }{ }^{\text {xviii, }} \text { libérer, } \\
\text { repousser... }\end{array}$ \\
\hline
\end{tabular}

i. Le site de Andreas Lutz (http://www.andreaslutz.com/) propose une navigation par webcam ou par micro.

Toutes les pages auxquelles ce tableau renvoie ont été consultées le 14 janvier 2011.

ii. Bannière publicitaire pour "The National Fondation for the Deaf", http://www.bannerblog.com.au/2009/09/nfd_lip_reading_lesson. php, 2009.

iii. http://www.bannerblog.com.au/2009/06/pringles.php.

iv. http://www.bannerblog.com.au/2009/09/fas_twitter.php.

v. Rafael Rozendaal, http://www.popcornpainting.com/, 2008, http:// www.hotdoom.com/, 2009

vi. http://www.red-issue.com/.

vii. Marie Bélisle, Scriptura et caetera, "Alter ego », http://www.scripturae. com/, 1998.

viii. Anonymes, tableau « défragmenter", http://www.anonymes.net/ anonymes.html. ix. Toucher, tableau "caresser", http://www.to-touch.com/, 2009.

x. Marie Bélisle, Scriptura et caetera, "Alter ego ", http://www.scripturae. com/, 1998.

xi. Sophie Calle, Vingt ans après, http://www.panoplie.org/ecart/calle/ calle.html/, 2001.

xii. http://soytuaire.labuat.com/

xiii. Rafael Rozendaal, http://www.hybridmoment.com/, 2009.

xiv. Bannière publicitaire pour Compania Athletica, http://www.

bannerblog.com.au/2009/07/cia tramp.php . Bannière publicitaire pour une voiture (Meriva), http://www.bannerblog.com.au/2009/09/ gm_meriva_drop.php.

xv. http://www.bannerblog.com.au/2009/07/samsung_led.php.

xvi. http://www.bannerblog.com.au/2009/09/commbank_finances.php.

xvii. Rafael Rozendaal, http://www.coldvoid.com/, 2008

xviii. http://www.bannerblog.com.au/2009/09/toilet.php. 


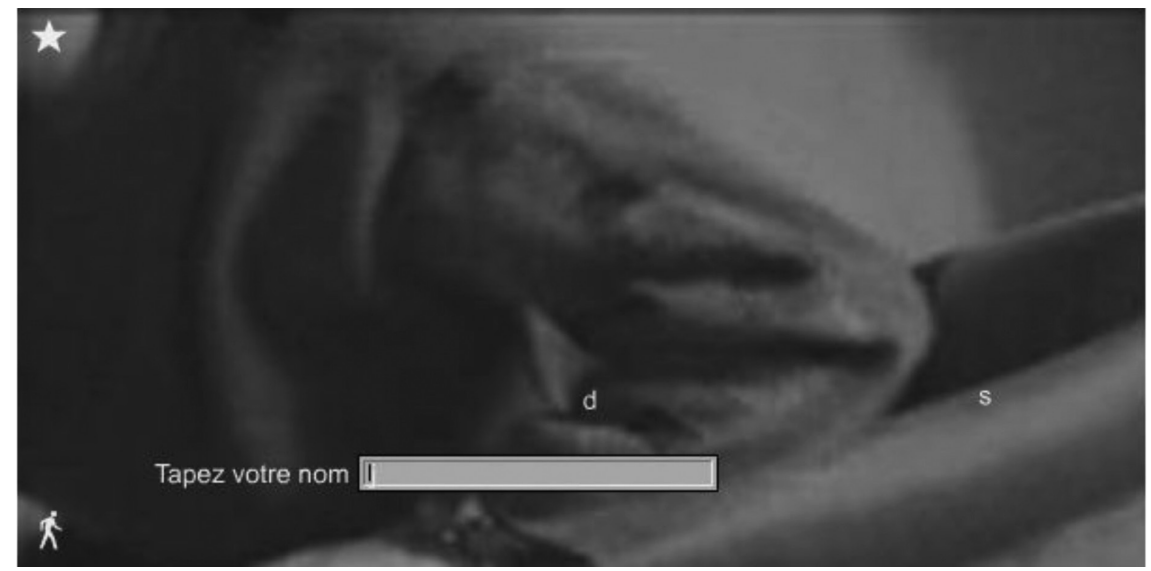

Figure 2

Anonymes version 1.0, tableau "Nom-dit" (2005): http://www.anonymes.net/anonymes.html

leur description en actèmes. Nous précisons si cette unité est délimitée ou non dans le temps et si elle est ou non répétitive. Les traits d'iconicité permettent d'insister sur la co-typie, à savoir le lien avec les expériences dans le monde physique. Précisons que ce tableau est le fruit d'un travail en cours et qu'il n'a aucune prétention à l'exhaustivité.

\section{FIGURES DE MANIPULATION DANS LA CRÉATION NUMÉRIQUE}

Nous avons vu que les unités sémiotiques de manipulation, en contexte, constituaient des couplages média. Ces couplages média donnent naissance à des figures. C'est la construction d'une telle figure que nous allons à présent analyser. La publicité mais aussi la littérature numérique et l'art numérique en ligne font en effet largement appel à ce qu'on peut appeler des figures de manipulation.

Prenons l'exemple de la création intitulée Anonymes version 1.0, et notamment du premier tableau dont le titre est "Nom-dit». Sur la page d'accueil, une zone réactive constituée par le texte "Anonymes» permet à l'internaute d'accéder à un premier tableau. Une vidéo tourne en boucle, représentant un homme qui se lève d'un fauteuil, apparemment pour ne pas être filmé (rester anonyme?). Un accompagnement sonore (des bruits de pas ou tout simplement le bruit de la vidéo?) joue en boucle également. Une fenêtre de saisie se présente à l'internaute accompagnée d'un texte:
«Tapez votre nom». Le tableau est donc en attente d'une action de l'internaute.

L'internaute tape une lettre, mais celle-ci, au lieu de rester dans la fenêtre de saisie, s' «envole» et disparaît de la fenêtre. L'internaute est tenté de taper à nouveau une lettre, notamment pour vérifier si le fonctionnement sera le même. La deuxième lettre s'envole également. L'internaute peut alors taper rapidement plusieurs lettres, tout un mot, pour voir les lettres dispersées dans l'espace du tableau. On aboutit à un résultat proche de certains poèmes cinétiques (lettres mises en espace et en mouvement), mais ici les lettres résultent d'une action (introduction de données au clavier) de l'utilisateur.

Le sens du tableau (les lettres du nom qui s'envolent pour disparaître, inéluctablement) est à mettre en rapport avec le titre de l'ensemble de la création, Anonymes. La figure qui résulte du geste (impossibilité de graver son nom) fait écho à la vidéo (un homme veut quitter le champ de la caméra) et peut-être également au son (bruit de pas qui s'éloignent?).

Ce tableau n'a pas de fin: éternellement nous resterons anonymes. L'internaute a néanmoins la possibilité de revenir à la page d'accueil (icône en haut à gauche) ou de passer au tableau suivant (icône en bas à gauche).

Pour reprendre notre terminologie, l’USM mobilisée consiste ici à «activer» (de façon ponctuelle, 
en appuyant sur des touches du clavier). Mais cette manipulation ne prend tout son sens que dans le couplage média, voire dans l'ensemble du discours interactif.

Nous avons bien ici un couplage non conventionnel avec le processus d'inscription à l'écran (par exemple l'envol des lettres) dans la mesure où le fonctionnement de la zone de saisie est détourné. Le fonctionnement d'une zone de saisie fait partie de l' «encyclopédie» (Klinkenberg, 2000) de l'utilisateur d'une interface informatisée (l'encyclopédie mobilisée dans le cas présent repose sur l'ergonomie des interfaces homme-machine et non sur une sémiotique linguistique). Une fenêtre de saisie permet conventionnellement de taper au clavier une chaîne de caractères, sans qu'ils ne disparaissent au fur et à mesure. Or, dans «Nom-dit», la fenêtre permet bien de saisir du texte, mais pas de le conserver. L'attente de l'utilisateur est troublée.

Analysons le processus de construction. La fenêtre de saisie, associée au texte "Tapez votre nom», suggère l'attente du geste de taper un caractère au clavier. Toutefois, quand on tape une lettre au clavier, on «inscrit» quelque chose qui, du moins c'est ce que l'on projette, va être conservé. Les champs de saisie dans lesquels on nous demande notre identité s'intitulent d'ailleurs souvent «s'inscrire» ou «s'enregistrer». Il est en outre intéressant de noter que, en certaines circonstances, ceux qui utilisent un traitement de texte pour la première fois ne comprennent pas pourquoi il faut enregistrer; pour eux, taper des lettres au clavier a valeur d'enregistrement. Les traits signifiants possibles liés à l'action (saisir une lettre au clavier) et au contexte média (la fenêtre de saisie associée au texte "Tapez votre nom») sont: inscription, enregistrement. Or, les lettres saisies s'envolent et disparaissent. Nous avons ici une suppression du trait signifiant possible «inscription» (durable) avec l'envol et la disparition de la lettre. La saisie des caractères est possible, mais pas l'inscription.

Nous faisons ici l'hypothèse que la construction d'une interprétation se fait notamment avec la répétition du geste. Le fonctionnement du couplage non conventionnel repose en effet ici sur la répétition. C'est en répétant le geste (ici, taper des lettres au clavier) que l'utilisateur construit progressivement le sens de la figure (et comprend qu'il ne s'agit pas d'un bogue). Il va pour cela prendre en compte l'ensemble des médias et de l'interface. Le média vidéo (+ son) permet alors de donner tout son sens à la figure. Font ainsi sens non seulement le contenu de la vidéo (un homme qui s'éloigne), mais également sa forme (boucle). Des traits signifiants possibles d'une boucle vidéo sont, en effet, circularité, éternel recommencement. Le trait signifiant de la boucle vidéo ("circularité») se superpose au trait signifiant «inscription» (adjonction puis suppression). La forme de la boucle est ici associée aux lettres qui disparaissent inéluctablement de la zone de saisie: prise dans une circularité, toute saisie ou toute inscription durable est impossible (on peut aussi considérer que l'inscription à l'écran se trouve démultipliée, mais elle n'a pas de pérennité). Nous avons ici une construction pluricode entre le trait signifiant «circularité» (boucle vidéo) et le trait «inscription» (geste couplé à la fenêtre de saisie). Le lecteur en tire une conséquence: «Je resterai anonyme».

D'autres interprétations sont possibles. Par exemple, si l'on se situe sur le plan de la création, on peut considérer que ce qui vole en éclats ici, c'est l'illusion de participer à l'œuvre. L'internaute ne peut le faire (cela dit, il participe néanmoins au visuel de l'œuvre, mais uniquement pour lui-même). Est mise en scène une réflexion sur l'interactivité et la participation du lecteur dans les ouvres dites contributives.

Dans un deuxième temps, il faudrait resituer ce tableau dans l'ensemble d'un discours, en prenant en compte certes l'interface liminaire, mais aussi l'ensemble des autres tableaux de cette «version $1.0 \%$. Sur l'écran d'accueil, on peut voir des abeilles sur fond de ruche tourner sans fin. Elles aussi sont anonymes au sein de la ruche. On peut accéder au premier tableau en cliquant sur l'alvéole de la ruche correspondante, ou bien en cliquant sur le titre de l'œuvre ("Anonymes»), qui constitue aussi une zone réactive (au survol s'affiche le texte "Entrer»). C'est ce contexte média d'interaction qu'il faut également prendre en compte dans l'analyse de cette figure. 


\section{CONCLUSION}

Pour étudier les créations numériques interactives et multimédias, nous avons besoin d'outils d'analyse sémiotique spécifiques. Le modèle en cinq niveaux présenté dans cet article a avant tout une vocation heuristique visant à mettre au jour les spécificités de la manipulation gestuelle dans une création numérique.

Le geste de manipulation, tout comme le mouvement pour l'animation, autorise des couplages conventionnels et non conventionnels avec différents médias. Ces couplages donnent lieu à des figures. Cet article s'inscrit dès lors dans une démarche de formalisation d'une rhétorique ${ }^{15}$ de l'écriture interactive. Nous pouvons en effet faire l'hypothèse de figures de rhétorique spécifiques à l'écriture interactive (Bouchardon, 2007). Il s'agit d'une catégorie de figures à part entière, à côté des figures de diction, de construction, de sens et de pensée, que l'on peut qualifier de «figures de manipulation". Deux points sont ici à souligner. D'une part, la notion de «figure» peut - et ceci est nouveau - prendre en compte le geste du lecteur. D'autre part, l'écriture interactive, plus que sur des figures de sens comme les tropes, s'appuie sur des figures de manipulation. De façon plus générale, cet article vise à apporter une contribution à la mise au jour de spécificités de l'écriture interactive.
* Tous les exemples en ligne cités dans cet article ont été consultés entre le 14 et le 17 janvier 2011.

\section{NOTES}

1. Exemple: http://www.bannerblog.com.au/2009/09/commbank_ finances.php.

2. Exemple: http://www.bannerblog.com.au/2009/09/fas_twitter. php.

3. Exemple: http://www.youtube.com/watch?v=4ba1BqJ4S2M. 4. Cette recherche aboutira en 2011 à un ouvrage intitulé Signes et figures de la création numérique. Ce travail repose sur un corpus de 100 bannières publicitaires en ligne et de vingt œuvres de littérature et d'art numériques.

5. Nous utilisons ici le terme d' «interactivité», même si Yves Jeanneret récuse la "métaphore de l'interactivité » comme faisant "obstacle, concrètement, à l'étude attentive des objets " (2000: 114).

6. Une taxonomie de gestes pour interagir sur un bureau 3D: http://gizmodo.com/5371913/bumptop-3d-desktop-gets-uniquemulti+touch-gestures?autoplay=true.

7. Le site "Publicis \& Hal Riney" (http://www.hrp.com/) propose une navigation par webcam.

8. Le site de Andreas Lutz (http://www.andreaslutz.com/) propose une navigation par webcam ou par micro.

9. Exemple: http://www.youtube.com/watch?v=p3QgigeSE1s. 10. Voir l'article d'Alexandra Saemmer dans ce même numéro. 11. On peut décomposer un mouvement continu (comme le déplacement d'une souris) en différents gestèmes. Ainsi, pour certains, le "mickey" va constituer l'unité de déplacement du pointeur de la souris équivalente à $1 / 200$ e de pouce, soit 8 pixels. Cette unité peut permettre de distinguer différents gestèmes dans la manipulation physique de la souris.

12. En ligne: http://www.newrafael.com/websites/.

13. Nous renvoyons à l'article d'Alexandra Saemmer dans ce même numéro pour des précisions concernant les couplages conventionnels et non conventionnels.

14. Ce dernier couplage autorise ce que Philippe Bootz appelle la "double lecture" (2004).

15. Nous renvoyons également à l'article d'Alexandra Saemmer dans ce même numéro pour une réflexion sur la notion de figure dans la création numérique.

16. La rhétorique classique comprend cinq grandes parties: l'invention, la disposition, l'élocution, la mémoire et l'action. L'élocution s'attache plus particulièrement à l'aspect littéraire et esthétique du discours. C'est ce que retiendra la «rhétorique restreinte». L'expression est de Michel Pougeoise : "Parmi les partisans de cette rhétorique que l'on pourrait qualifier de "littéraire" car elle s'intéresse essentiellement à l'étude des figures et du style, il faut citer J. Cohen, G. Genette, H. Morier ainsi que le groupe $\mu$ " (2001: 203-204). C'est à cette rhétorique restreinte que nous nous intéressons ici : poser la question d'une rhétorique de l'écriture interactive, c'est poser la question d'une spécificité de cette écriture en termes de style et notamment de figures. 


\section{RÉFÉRENCES BIBLIOGRAPHIQUES}

AARSETH, E. [1997]: Cybertext, Perspective on Ergodic Literature, Baltimore, John Hopkins University Press.

BoOTZ, P. [2004] : «der/die Leser/Reader/Readers", dans F.W. Block, C. Heibach et K. Wenz (dir.), p0es1s. Ästhetik digitaler Poesie/The Aesthetics of Digital Poetry, Ostfildern-Ruit, Hatje Cantz Verlag, 936-122, [2005]: Le Rabot_poète. En ligne: http://www.sitec.fr/users/ akenatondocks/DOCKS-datas_f/collect_f/auteurs_f/B_f/BOOTZ_F/ Animations_F/rabot.htm.

BOUCHARDON, S. [2007]: «L'écriture interactive: une rhétorique de la manipulation", dans I. Saleh I. et alii (dir.), Collaborer, échanger, inventer: expériences de réseaux, Actes de H2PTM'0, Paris, Hermès-Lavoisier, $155-170$;

[2008]: «Une esthétique de la matérialité», dans A. Saemmer et M. Maza (dir.), E-formes. Écritures visuelles sur supports numériques, SaintÉtienne, Publications de l'Université de Saint-Étienne, 135-144;

_ [2009]: Littérature numérique : le récit interactif, Paris, Hermès-

Lavoisier.

DALLET, J.-M. [1996]: «Quelques éléments d'une grammaire du geste interactif ", Artifices 4. En ligne: http://www.ciren.org/artifice/ artifices 4/dallet.html.

GHITALlA, F., D. BOUlLIER et alii [2004]: L'Outre-lecture: manipuler, (s') approprier, interpréter le Web, Paris, Éd. du Centre Georges-Pompidou. JEANNERET, Y. [2000]: Y a-t-il vraiment des technologies de l'information?, Paris, Éd. universitaires du Septentrion.

Klinkenberg, J.-M. [(1996) 2000]: Précis de sémiotique générale, Paris, Seuil.

Pougeoise, M. [2001]: Dictionnaire de rhétorique, Paris, Armand Colin. Queneau, R. [1961]: Cent mille milliards de poèmes, Paris, Gallimard. SAEMMER, A. [2007]: Matières textuelles sur support numérique, SaintÉtienne, Publications de l'Université de Saint-Étienne;

[2008]: «Le texte résiste-t-il à l'hypermédia?», Communication et Langages, no 155, Paris, Armand Colin, 63-79.

WeissberG, J.-L. [2006], "Corps à corps - À propos de La Morsure, CD ROM d'Andréa Davidson", dans P. Barboza et J.-L. Weissberg (dir.),

L'Image actée. Scénarisations numériques : parcours du séminaire L'action sur l'image, Paris, L'Harmattan, 51-71.

Wobbrock, J. O., M. Ringel Morris et A. D. Wilson [2009]: User Defined Gestures for Surface Computing, CHI2009. En ligne: http://dub. washington.edu/pubs/144. 